\title{
Training and fairer payments would increase caries prevention in practice
}

\author{
Abstracted from \\ Factors that drive dentists towards or away from dental caries preventive measures: \\ systematic review and metasummary. PLoS One 2014; 9: e107831. \\ doi: 10.1371/journal.pone.0107831. eCollection 2014. PubMed PMID: 25296335; \\ Address for correspondence: RS Terada, Department of Dentistry, State University of Maringá, \\ Maringá, Brazil. E-mail: rssterada@uem.br
}

Suga US, Terada RS, Ubaldini AL, Fujimaki M, Pascotto RC, Batilana AP, Pietrobon R, Vissoci JR, Rodrigues CG.

\section{Question: What factors determine whether dentists are more or less likely to provide caries prevention for their patients?}

Data sources PubMed, Embase, Psycolnfo, Scielo, Scopus, Web of Science, BBO, Lilacs and York databases supplemented by Google scholar.

Study selection Qualitative studies with findings derived from individual or group interviews and structured questionnaires reporting factors that drove dentists towards or away from incorporating caries preventive measures were considered. Two reviewers conducted selection independently.

Data extraction and synthesis Data were abstracted independently by two reviewers and a qualitative metasummary carried out. This involves the extraction, grouping and formatting of findings and the calculation of frequency and intensity effect sizes in order to provide mixed research syntheses and to conduct a posteriori analyses of the relationship between reports and findings.

Results Seven qualitative studies and 41 surveys $(36,501$

participants) were included. The reports were grouped together into six categories that were judged to be topically similar; education and training, personal beliefs, work conditions, remuneration, gender, place of residence and patients. Biologicism (27\%), low remuneration (25\%), length of time since graduation (22\%) and male dentists (19\%) were found to be the highest frequency effect size driving dentists away from using preventive approaches, and team work (21\%), post-graduation (12\%) and professional understanding of the benefits (12\%) were identified as the main reasons for dentists' adherence to preventive measures.

Conclusions Despite the questionable quality of the included reports the evidence that emerged seems to indicate that further education and training coupled with a fairer pay scheme would be a reasonable approach to change the balance in favour of the provision of dental caries preventive measures by dentists. The results of this review could be of value in the planning and decision-making processes aimed at encouraging changes in professional dental practice that could result in the improvement of the oral health care provided to the population in general.

\section{Commentary}

Qualitative research is used with ever more increasing frequency in dentistry to 'identify the thoughts, feelings, attitudes, perceptions and preferences of people'. ${ }^{1}$ Qualitative reviews of any sort, however, are still few and far between and generally considered to be an expanding and evolving methodological area. ${ }^{2}$ The results from qualitative studies can be difficult to generalise from even when the data collection has been carried out in similar ways from similar groups of individuals. ${ }^{3}$ Tools such as the ENTREQ statement, designed to 'enhance transparency in reporting the synthesis of qualitative data' ${ }^{2}$ are useful when appraising purely qualitative reviews. The ENTREQ statement is made up of twenty-one items each with a guide and description which enable reporting of solely qualitative syntheses. ${ }^{2}$ A mixed-methods review is one way of combining the results from both qualitative and quantitative studies with a view to 'maximising findings to inform policy and practice'. ${ }^{4}$ One example of a mixed-methods review approach is the Evidence for Policy and Practice Information (EPPI) review which allows diverse sources of evidence to be appraised and combined though parallel synthesis and reporting. ${ }^{4}$ The systematic review described in this article combines the results of both qualitative and quantitative studies although a formal mixed-methods protocol does not appear to have been used to synthesise the findings.

The aim of this systematic review and metasummary is to 'analyse studies that have investigated the factors that drive dentists towards or away from dental caries preventative measures'. The research question is not explicitly defined within the article although is inferred within the aims section and main title. The reviewers refer to the PRISMA statement for systematic reviews and meta-analyses ${ }^{5}$ which is generally used when combining data from purely quantitative studies. This statement does, however, provide a reasonable framework on which to base the systematic search for any type of study.

The reviewers use a comprehensive approach to searching by looking to find all available studies under the terms listed within their search strategy. A contrasting iterative search approach is sometimes used within qualitative research where 'all available concepts are sought until theoretical saturation has been achieved'. ${ }^{4}$ The inclusion criteria of the review are clearly stated and include qualitative studies of all types and surveys with structured questionnaires. The review participants are limited to dentists and so studies which report responses from other members of the dental team are not included. The authors exclude studies which present findings relating to clinical preventative measures such as fluoride varnish 
application and fissure sealants. Specific types of preventative measures which were included (eg tooth brushing, dietary advice etc) are not defined.

The authors search a variety of data sources including a large number of appropriate electronic databases (PubMed, Embase, PsychInfo, Sciclo, Scopus, Web of Science, BBO, Lilacs and York) as well as crosschecking the reference lists of identified studies and contacting experts on the topic to identify areas of grey literature. This search is unrestricted in terms of language filters and year limits. A clear electronic search strategy is provided which follows that of a traditional systematic review. The study screening methods are described in full with two reviewers independently retrieving titles, abstracts and full text articles. A third reviewer was available to assist with achieving consensus agreement on the articles to be included. The study characteristics for each of the included studies are presented within two tables. These are split into two categories; those defined as 'qualitative studies' and those described as 'surveys'. Listed authors, sample size, type of study (described as intervention within the table) and objectives plus outcome of the study are all included. Seven qualitative studies, reflecting the views of 390 participants, and 41 survey type studies $(36,111$ participants in total) are included. The study selection results are presented as a CONSORT ${ }^{8}$ type flow diagram showing the number of publications identified, retrieved, extracted and included in the final analysis.

Quality assessment in qualitative research has been described as being especially challenging. ${ }^{2,3}$ The authors describe the quality assessment criteria and process used in full. They chose to appraise the qualitative and quantitative studies separately and make use of two tools to do this; the Joanna Briggs Institute Qualitative Assessment and Review ${ }^{6}$ and items adapted from Reporting Guidelines for Survey Research by Bennett et al..$^{7}$ It is not described within the paper whether the appraisal process was conducted independently, by more than one author, or that consensus was agreed. The quality appraisal is not without limitations, as it appears to be an accumulative type scoring system with potential to allow areas of good quality within a study design to compensate for those of lesser quality. Based on the prevalence of 'yes' and 'no' answers to questions about quality appraisal items the results are included in the risk of bias as low, moderate or high. A heat map showing the gradient of quality indicators for both the surveys and qualitative studies was produced. No weighting was carried out and no articles were excluded on the basis of the quality assessment. The calculated quality score for each of the papers reviewed is not provided. Risk of bias assessment is included in results tables with description of each study.

The reviewers develop categories of factors which drive dentists towards or away from engaging in preventative strategies with their patients. Qualitative data analysis software (ATLAS. Ti7) was used to codify themes and build a metasummary of both the qualitative and quantitative findings. A metasummary is defined as 'a quantitatively orientated aggregation of qualitative findings originally developed to accommodate the different characteristics of qualitative studies and surveys'. ${ }^{9}$

In this summary the process of deriving themes was inductive and carried out in such a way as to 'quantify the qualitative data'. ${ }^{4}$ The relative magnitude of the extracted results was calculated giving the percentage of studies which mentioned each category of barrier or enabler to providing caries preventative measures. The analysis was undertaken by two reviewers although the specific process for coding within each study is not described. The main themes derived include; education and training, personal beliefs, work conditions, remuneration, gender, place of residence and patient factors.

Whilst in keeping with the term 'metasummary', ${ }^{9}$ the synthesis output does not really go beyond a summary of the primary findings. Pope et al. describe syntheses of qualitative with quantitative evidence as recent endeavours which are few and far between, and generally draw on the methods and analytical techniques used within qualitative studies. ${ }^{4}$ However, quotations are not provided within this review paper. Six of the studies explore the attitudes, beliefs and motivations for caries prevention in children although this is not explored further within the descriptive body of the review. Individual studies relate to a wide range of topics from dental contract implementation to the use of specific preventative agents. The review finds that dental education and training is an important factor in influencing dentists' drive towards preventative treatments for caries, with remuneration systems also suggested as having a strong influence.

The authors of this article have provided a structured systematic review search strategy and a metasummary of results. There are some limitations with regard to the quality of the evidence retrieved and summary strategy used. The review may have benefitted from the use of a recognised mixed method protocol (such as $\mathrm{EPPI}^{4}$ ) and through a clearer definition of the preventative measures being included. In doing so they could have truly synthesised the findings from both study types and maximised the impact of the results. Nevertheless, the authors bring to the attention of the dental research community the difficulties and limitations of carrying out a mixed-methods type review which includes qualitative and quantitative dental data. This is an area which warrants development in order to make the most of the breadth of studies available for metasummary and synthesis.

\section{Susan J Carson and Ruth Freeman Dental Health Services Research Unit, School of Dentistry, University of Dundee, Dundee, Scotland, UK}

1. Stewart K, Gill P, Chadwick B, Treasure E. Qualitative research in dentistry. Br Dent J 2008; 204: 235-239. doi:10.1038/bdj.2008.149.

2. Tong A, Flemming K, Mclnnes E, Oliver S and Craig J. Enhancing transparency in reporting the synthesis of qualitative research: ENTREQ. BMC Med Res Methodol 2012; 12: 181. doi:10.1186/1471-2288-12-181.

3. Pope C, Mays N. Qualitative research in health care. Oxford: Blackwell Publishing, 2006. 4. Pope C, Mays N, Popay J. Synthesizing Qualitative and Quantitative Health Evidence. Maidenhead: Open University Press, 2007.

5. Moher D, Liberati A, Tetzlaff J, Altman DG; PRISMA Group (2009). Preferred reporting items for systematic reviews and meta-analyses: the PRISMA statement. PLoS Med 2009; 6: e1000097.doi:10.1371/journal.pmed.1000097.

6. Joanna Briggs Institute Reviewers' Manual: 2014 edition. Available online at: http:// joannabriggs.org/assets/docs/sumari/reviewersmanual-2014.pdf [accessed 27th February 2015].

7. Bennett $C$, Khangura S, Brehaut JC, et al. Reporting guidelines for survey research: an analysis of published guidance and reporting practices. PLOS Med 2010; 8: e1001069. doi:10.1371/journal.pmed.1001069. Epub 2011 Aug 2

8. Schulz KF, Altman DG, Moher D; CONSORT Group. CONSORT 2010 Statement: updated guidelines for reporting parallel group randomised trials. BMC Med 2010 8: 18. doi:10.1186/1741-7015-8-18. Available on line at: http://www.consortstatement.org/Media/Default/Downloads/CONSORT\%202010\%20Statement/ CONSORT\%202010\%20Statement\%20-\%20BMC\%20Medicine.pdf [accesses 1st March 2015].

9. Sandelowski M, Barroso I, Voils $\mathrm{Cl}$. Using qualitative metasummary to synthesize qualitative and quantitative descriptive findings. Res Nurs Health 2007; 30: 99-111.

Evidence-Based Dentistry (2014) 16, 6-7. doi:10.1038/sj.ebd.6401072 